\title{
Patient Experience with Congenital (Hereditary) Thrombotic Thrombocytopenic Purpura: A Conceptual Framework of Symptoms and Impacts
}

\author{
Abiola O. Oladapo ${ }^{1} \cdot$ Diane Ito $^{1} \cdot$ Christopher Hibbard $^{1} \cdot$ Stephanie E. Bean ${ }^{2} \cdot$ Robert N. Krupnick $^{3}$. \\ Bruce M. Ewenstein ${ }^{1}$
}

Published online: 30 July 2019

(C) Takeda 2019

\begin{abstract}
Background and Objective Thrombotic thrombocytopenic purpura is a rare, life-threatening disorder characterized by microangiopathic hemolytic anemia and thrombocytopenia, with variable clinical manifestations (e.g., central nervous system, renal, gastrointestinal, and cardiac effects). This study's objective was to gain an in-depth understanding of patients' experiences with the congenital form of thrombotic thrombocytopenic purpura, including the most salient symptoms and impacts associated with congenital thrombotic thrombocytopenic purpura and its treatment.

Methods An initial conceptual model of thrombotic thrombocytopenic purpura symptoms and impacts was derived from a targeted literature review, refined by interviews with expert hematologists, and further refined by concept elicitation telephone interviews with adults with congenital thrombotic thrombocytopenic purpura in the USA. Patients reported the duration, frequency, and severity experienced for each concept, and rated level of disturbance on a minimum to maximum scale of 0-10. Results Interviews were conducted with 11 patients (mean age, 38.2 years; range 21-52 years) in three waves ( $n=4, n=4$, $n=3$ ). The most salient symptoms (reported most frequently and rated by patients as most disturbing) were fatigue, headache, bruising, joint pain, muscular pain, forgetfulness, and difficulty communicating. The most salient impacts included diminished ability to work/study, financial distress, feeling depressed, feeling anxious, and mood swings. Patients' comments reflected the pervasive nature of congenital thrombotic thrombocytopenic purpura symptoms and impacts, and unmet treatment needs. Conclusions The final conceptual model, which includes salient symptoms and impacts of congenital thrombotic thrombocytopenic purpura and reflects the disease burden, was derived by integrating inputs from the literature review, expert opinion, and patient interviews, and will be used to develop a congenital thrombotic thrombocytopenic purpura-specific, patient-reported outcome instrument.
\end{abstract}

\section{Introduction}

Thrombotic thrombocytopenic purpura (TTP) is a rare, life-threatening disorder of the blood coagulation system. The incidence of TTP is approximately two to ten cases per

Electronic supplementary material The online version of this article (https://doi.org/10.1007/s40271-019-00365-y) contains supplementary material, which is available to authorized users.

Abiola O. Oladapo

Abiola.oladapo@takeda.com

1 Baxalta US Inc., A Member of the Takeda Group of Companies, Cambridge, MA, USA

2 IQVIA, New York, NY, USA

3 IQVIA, Cambridge, MA, USA million per year and can be congenital (cTTP) or acquired (aTTP, also referred to as immune-mediated TTP), with cTTP accounting for $5-10 \%$ of cases [1-3]. The prevalence of cTTP is less than one case per million [3].

Patients with cTTP experience enhanced platelet aggregation, partial vessel occlusion by platelet-rich thrombi $[1,4]$, thrombocytopenia, and multi-organ dysfunction as a result of widespread microvascular ischemia [1, 4, 5]. If untreated, acute TTP episodes are associated with high morbidity and mortality, necessitating prompt diagnosis and treatment. Potential disease-related complications include stroke and persistent renal or neurologic abnormalities [6]. It is recognized that cognitive decline is a major complication for patients with TTP, which impacts their concentration, information processing, rapid language generation, and memory performance, thus impacting their work productivity [7]. 


\section{Key Points for Decision Makers}

The symptoms and impacts of congenital thrombotic thrombocytopenic purpura were evaluated by a literature review and interviews with hematologists and patients through a process in accordance with industry and research best practice guidelines.

The most salient symptoms of congenital thrombotic thrombocytopenic purpura were fatigue, headache, bruising, pain in joints, muscular pain, forgetfulness, and difficulty communicating; the most salient impacts included diminished ability to work/study, financial distress, feeling depressed, feeling anxious, and mood swings.

These findings will be used to develop a congenital thrombotic thrombocytopenic purpura-specific, patientreported outcome instrument that may be useful to assess outcomes of treatment in clinical practice or in future clinical trials.

Many patients with cTTP require ongoing prophylactic treatment with plasma infusion (e.g., every $2-3$ weeks or before triggering situations) $[1,8]$. Prophylaxis schedules are determined empirically, with the intensity of treatment depending on symptoms, the presence of triggering factors, and patient preference $[1,9]$. Once initiated, most patients require prophylactic plasma infusions for life, which entail intravenous access and travel to a facility at regular intervals [9]. In addition, other treatment-related adverse events and limitations may further contribute to patient discomfort and distress, and for patients who do not live close to infusion centers, the need to travel regularly for treatment is an inconvenience to them and their caregivers and contributes to the overall disease burden $[6,10]$. Currently, direct patient reports of the impacts of cTTP are lacking, especially as they relate to patients' experiences of living with the disease. Given this is a rare disease, little is known about the overall disease burden and impacts of cTTP, including the effects on mood and health-related quality of life.

The objective of this study was to gain an in-depth understanding of the patient experience with cTTP, including the most salient symptoms of cTTP and the words and phrases patients use to describe the disorder. We hypothesized that the symptoms of cTTP would have a profound effect on patients' quality of life. The goal was to develop a final conceptual model capturing the symptoms and impacts of cTTP that may be useful when evaluating treatment outcomes, and in the development of a patient-reported outcome (PRO) instrument that reflects the experience of patients with the disorder.

\section{Methods}

\subsection{Literature Review}

A targeted literature review was first conducted to construct a preliminary conceptual model of the symptoms and impacts of cTTP. Database searches for English-language articles were conducted in PubMed, Cochrane, PsychInfo, ScienceDirect, American Society of Clinical Oncology, American Society of Hematology, and Access Medicine from the inception of each database through March 2016. Search strategy terms included thrombocytopenic purpura, TTP, cTTP, hTTP, aTTP, Upshaw-Schulman Syndrome, USS, symptoms, quality of life, and review. The searches were intended to be broad and all-inclusive in terms of the types of articles. Google searches were also performed to identify experiences reported by adult patients or patient groups with TTP (including patient blogs) based on the above search terms AND/OR "patient(s') experience." An initial conceptual model was derived from the literature review, accounting for signs, symptoms, immediate (direct) impacts, and general (indirect) impacts.

\subsection{Clinician Interviews}

Five experts in providing clinical management of patients with cTTP in the USA, the UK, and Austria, were interviewed to understand clinician perspectives and to test the initial conceptual model derived from the literature review. Interviews were conducted (July 2016) by telephone and screen sharing, and guides were used to ensure all clinicians were asked the same questions. Clinicians were first asked open-ended questions about their perspectives on the signs, symptoms, and impacts of cTTP, before providing input into how the model generated from the literature review could be modified and improved.

\subsection{Patient Interviews}

In a rare disease such as cTTP, obtaining a sample of patients who are a representative subset of the larger patient population can be quite challenging. Two recruiting sources were employed to identify as many of these rare patients as possible to serve the specific needs of this research. First, a third-party vendor specialized in recruiting patients with rare diseases solicited patients through non-profit organizations and by direct contact through the vendor's proprietary networking process. All ten patients identified by this vendor were accepted as possible candidates for inclusion in the study and were subsequently interviewed. In addition, a patient advocacy organization (Answering T.T.P.-Thrombotic Thrombocytopenic Purpura Foundation) was engaged 
and posted information about the study on its website and Facebook page. The organization's request yielded three interested patients, one of whom was interviewed, one who declined at the time of interview because of an exacerbating illness, and one who did not respond to scheduling after repeated attempts.

The study was performed in accordance with International Society for Pharmacoeconomics and Outcomes Research Good Research Practice guidance for eliciting concepts for PRO instruments: (1) determine the context of use; (2) develop the research protocol for qualitative concept elicitation and analysis; (3) conduct the concept elicitation interviews or focus groups; (4) analyze the qualitative data; and (5) document concept development and elicitation [11, 12]. This aligned with the US Food and Drug Administration guidance for industry on PRO measures (2009) [13]. The protocol, informed consent language, and relevant supporting information were approved by a local institutional review board. Participants provided written informed consent with verbal confirmation of consent before initiation of each interview.

\subsection{Patient Concept Elicitation}

Inclusion criteria for patient eligibility included a diagnosis of cTTP, residence in continental USA, ability to speak English, and a willingness and ability to sign an informed consent form. Patients were excluded from the study if they had a history of either significant neurological events (such as major stroke) or a mental health condition rendering them unable to understand the nature, scope, and possible consequences of the study.

Patient concept elicitation interviews were conducted by two of the authors, one was male (RNK) and one was female (SEB). Interview duration was $60 \mathrm{~min}$, on average. The interview guide consisted of a standard set of openended and probing questions to assess participant experiences of cTTP. Probes were used to confirm or disconfirm the experience of symptoms and impacts in the conceptual model that were not contributed by the patient during the open-ended questioning.

Patients were asked about their first experience with cTTP, changes over time, and current signs, symptoms, and impacts of the condition and its treatments. Patients described the duration, frequency, and severity of currently occurring symptoms and impacts, and rated the levels of disturbance on a 0 (not at all) to 10 (greatly) scale. In eight of the 11 interviews when the respondent permitted sufficient time for deeper probing to augment understanding of the cTTP patient profile, patients were also asked to describe other aspects of their experience with cTTP, including body part(s) affected by symptoms (as appropriate), time of day when symptoms were most bothersome, factors that contributed to improvement or worsening of symptoms, and possible relationships between specific symptoms and impacts.

The interview schedule was designed to allow the guide to be updated and to assess concept saturation after a determined number of interviews were completed. Saturation is defined as the point after which no additional relevant concepts are elicited as interviews progress over time. In accordance with guidance for managing concepts originating late in the process, scientific judgment based on knowledge of the field was used to determine whether additional data collection was warranted after a determined number of interviews had been completed. Repeat interviews were not performed.

\subsection{Analysis of Data}

Content analysis was selected to apply an objective, systematic, and quantitative description of content communication. Coding was performed by a single data coder to identify and categorize concepts (signs, symptoms, and impacts) that are most important and relevant to patients. No formal coding tree was defined and no specific themes were defined in advance. However, the symptoms and impacts listened for and probed on were driven by the conceptual model derived from the initial literature review and interviews with clinicians (i.e., they were hypotheses for testing with patients).

As part of this process, patient statements reflecting similar concepts were grouped under the same theme at the analyst's discretion. In cases in which this categorization was unclear, the research team decided whether the symptom or impact should be counted separately, grouped within an existing theme, or if the wording of the existing theme should be changed. A distinction was made between concepts (symptoms and impacts) considered most salient by patients and those that are reported less frequently and/or are less disturbing to their lives. ATLAS.ti qualitative data analysis software was employed to assist with the content analysis of interview transcripts [14]. Once the coding process was completed, ATLAS.ti output listing the number of occurrences of each code was generated and served as the basis for summary tables of results.

\section{Results}

\subsection{Literature Review}

The literature review revealed articles that included general disease background references, diagnostic and treatment guidelines, as well as the development and clinical use of clinical outcome assessment instruments (typically PROs) used to measure symptoms and impacts of TTP and its variants. In general, the reviewed literature did not distinguish between symptoms of cTTP and aTTP. 
The most common signs of adult TTP discovered in the published literature (12 articles) [4, 9, 10, 15-23] included thrombocytopenia, microangiopathic hemolytic anemia, neurologic symptoms, renal failure, and fever. Key symptoms included bruising, chest pain, confusion, fatigue, general malaise, headache, jaundice, pallor, partial paralysis/ muscular weakness, red/purple spots on the skin, and vision problems. Headaches and abdominal pain were reported more often in association with cTTP, whereas tiredness was reported more often in patients with aTTP. Treatment-related effects included rash or hives, injection-site pain or discomfort, and side effects associated with steroid treatment (for patients with aTTP).

Thrombotic thrombocytopenic purpura impacts were grouped into one of two categories: immediate or general. Immediate impacts were defined as consequences of one or more disease symptoms that developed in close chronological proximity to symptom onset or rapid progression, or as consequences of treatment of those symptoms. General impacts were those more distal to the signs and symptoms, or their treatment, and considered to be less directly related; these resulted from accumulated experience with one or more symptoms over time. The most common immediate impacts included heart failure, seizure, and stroke. Other reported immediate impacts included blood in urine, coma, coughing up blood, gastrointestinal bleeding, menorrhagia, and retinal hemorrhage. The most commonly reported general impacts were travel burden and treatment burden, which included intravenous access and frequent travel to a facility [9]. The initial conceptual model of TTP that resulted from the literature review is shown in Fig. 1.

\subsection{Clinician Interviews}

Expert clinician interviews largely confirmed the initial literature-based conceptual model. Experts identified the most salient symptoms of cTTP affecting patients as fatigue, headache, general malaise, and difficulty in verbal expression. The most prominent general impacts noted were treatment and travel burden, and emotional consequences of cTTP (e.g., anxiety and depression). Expert interviews also suggested the deletion of some effects that were identified during the literature review but were viewed as less common by these clinicians, including partial paralysis and hypotension. In addition, the expert clinicians acknowledged the occurrence of heart failure, gastrointestinal bleeding, and coughing up blood, but suggested these were relatively less common experiences. These clinicians also noted that symptoms and impacts differ between cTTP and aTTP to a modest degree. They indicated that patients with aTTP experience symptoms in sudden acute episodes and face short-term treatment impacts, while those with cTTP experience more chronic effects. The more chronic nature of cTTP elevates the symptom of headaches as more relevant and salient than in aTTP, while the more frequent need for treatment elevates the impacts of travel and treatment burden as more salient for cTTP.

Clinicians also considered the signs, symptoms, and impacts of cTTP to be largely similar across adult and adolescent populations. Minor differences in the impacts associated with cTTP may reflect different lifestyles of adolescents and adults. For example, "financial stress" may be less pronounced among adolescent patients, who are more likely to attend school and to live with adult family members who are largely responsible for their financial well-being.

\subsection{Domains and Themes Identified by Patient Interviews}

Concept elicitation interviews were conducted during August-September 2016 with 11 adult patients ( $\geq 18$ years of age) diagnosed with cTTP. The interviews were designed to confirm the symptoms and impacts that are most frequently occurring and most disturbing to the lives of patients with cTTP, characterize the patient experience, and further develop the conceptual model. Patients were interviewed individually by telephone; in one case, a patient's husband joined the interview at the patient's request. Participants were aware of the interviewer's name, affiliation with IQVIA, and role as a researcher acting on behalf of a pharmaceutical client. Interviews were performed in three waves $(n=4, n=4, n=3)$.

Characteristics of the patient sample were as follows: eight were female, three were male; age range was 21-52 years, mean age was 38.2 years; and mean age at diagnosis was 27.5 years (ranged from "early teens" to age 40 years). The relatively late mean age at diagnosis appears to reflect several factors, most prominently including years of misdiagnosis. At the time of the interview, five of the 11 patients were undergoing continuous treatment regimens with prophylactic plasma infusion, while six patients received plasma as needed. Patient demographics for the interviewed sample were consistent with demographic characteristics of adult patients with cTTP described in the published literature [24, 25]. Based on commonly reported aspects of their experience, a composite vignette is provided in Box 1 of the Online Appendix.

For the purposes of organizing the data and possibly providing a framework for future instrument design and scoring, symptoms of cTTP were grouped into eight categories: energy, vision, blood pressure, pain, cognitive effects, side effects, treatment-related effects, and other. Mapping frequency of occurrence against degree of life disturbance, symptoms with relatively high frequency (mentioned by at least half of the patients interviewed) and high disturbance scores (rated by patients on average above " 5 " on a scale 
Fig. 1 Preliminary conceptual model for adult thrombotic thrombocytopenic purpura (TTP) developed following a comprehensive literature review

\begin{tabular}{|c|c|c|}
\hline $\begin{array}{l}\text { Patient Population } \\
\text { Adult patients } \\
\text { with TTP }\end{array}$ & \multicolumn{2}{|c|}{$\begin{array}{l}\text { Disease Process } \\
\text { Systemic platelet clumping in the microvascular and } \\
\text { small arterioles, resulting in thrombocytopenia and } \\
\text { microangiopathic hemolytic anemia }\end{array}$} \\
\hline \multicolumn{2}{|c|}{$\begin{array}{ll}\text { Signs and Symptoms of the Disease } \\
\text { - Thrombocytopenia } & \text { - Red/purple spots } \\
\text { - MAHA } & \text { on the skin } \\
\text { - Neurologic symptoms } & \text { - Vision problems } \\
\text { - Renal failure } & \text { - Abdominal pain } \\
\text { - Fever } & \text { - Aphasia } \\
\text { - Bruising } & \text { - Arthralgia } \\
\text { - Chest pain } & \text { - Dyspnea } \\
\text { - Confusion } & \text { - Hypotension } \\
\text { - Fatigue* } & \text { - Hypertension } \\
\text { - General malaise } & \text { - Myalgia } \\
\text { - Headache } & \\
\text { - Jaundice } & \text { - Nausea/vomiting } \\
\text { - Pallor } & \end{array}$} & $\begin{array}{l}\text { Treatment-Related } \\
\text { Effects } \\
\text { - Rash/hives } \\
\text { - Pain/discomfort at } \\
\text { injection site } \\
\text { - Steroid side effects }\end{array}$ \\
\hline $\begin{array}{l}\text { Immediate Impact } \\
\text { - Heart failure } \\
\text { - Seizure } \\
\text { - Stroke } \\
\text { - Blood in urine } \\
\text { - Coma } \\
\text { - Coughing blood } \\
\text { - Gastrointestinal bleeding } \\
\text { - Menorrhagia } \\
\text { - Retinal hemorrhage }\end{array}$ & $\begin{array}{l}\text { General Impact } \\
\text { - Travel burden } \\
\text { - Treatment burde } \\
\text { - Reduced ability to } \\
\text { - Anxiety } \\
\text { - Depression } \\
\text { - Financial distress } \\
\text { - Mood swings } \\
\text { - Negative physical } \\
\text { - Negative physical }\end{array}$ & $\begin{array}{l}\text { rk/study } \\
\text { ception by others } \\
\text {-perception }\end{array}$ \\
\hline
\end{tabular}

Items in boldface indicate the most salient symptoms and impacts MAHA = microangiopathic hemolytic anemia *More common in aTTP; ${ }^{\dagger}$ More common in CTTP from 0 to 10 ) in combination were considered to be most salient for patients with cTTP. As shown in Fig. 2, the most salient symptoms were fatigue, headache, bruising, pain in joints, muscular pain, forgetfulness, and difficulty communicating. Patient comments reflecting the pervasive and pernicious nature of some of these cTTP symptoms are summarized in Table 1.

Impacts of cTTP were grouped into five categories: emotional, activity restrictions, work/study restrictions, bleeding, and other physical impacts. As with symptoms, each cTTP impact was tabulated according to frequency and disturbance. Impacts with both high frequency and high disturbance were considered to be the most salient. As shown in Fig. 3, emotional impacts, including feelings of depression and anxiety, mood swings, and financial distress, were among the most frequently reported and most disturbing. Decreased ability to work or study was reported more often, but at a somewhat lower level of disturbance, on average. Comments from patients that illustrate some of these impacts of cTTP are presented in Table 1. In the context of discussing treatment-related impacts, patients consistently expressed a desire for safer and more convenient options (Table S1 of the Online Appendix).

Concept saturation for both symptoms and impacts was nearly $100 \%$, with all of the most salient concepts reaching the ideal full saturation objective. Saturation tables 


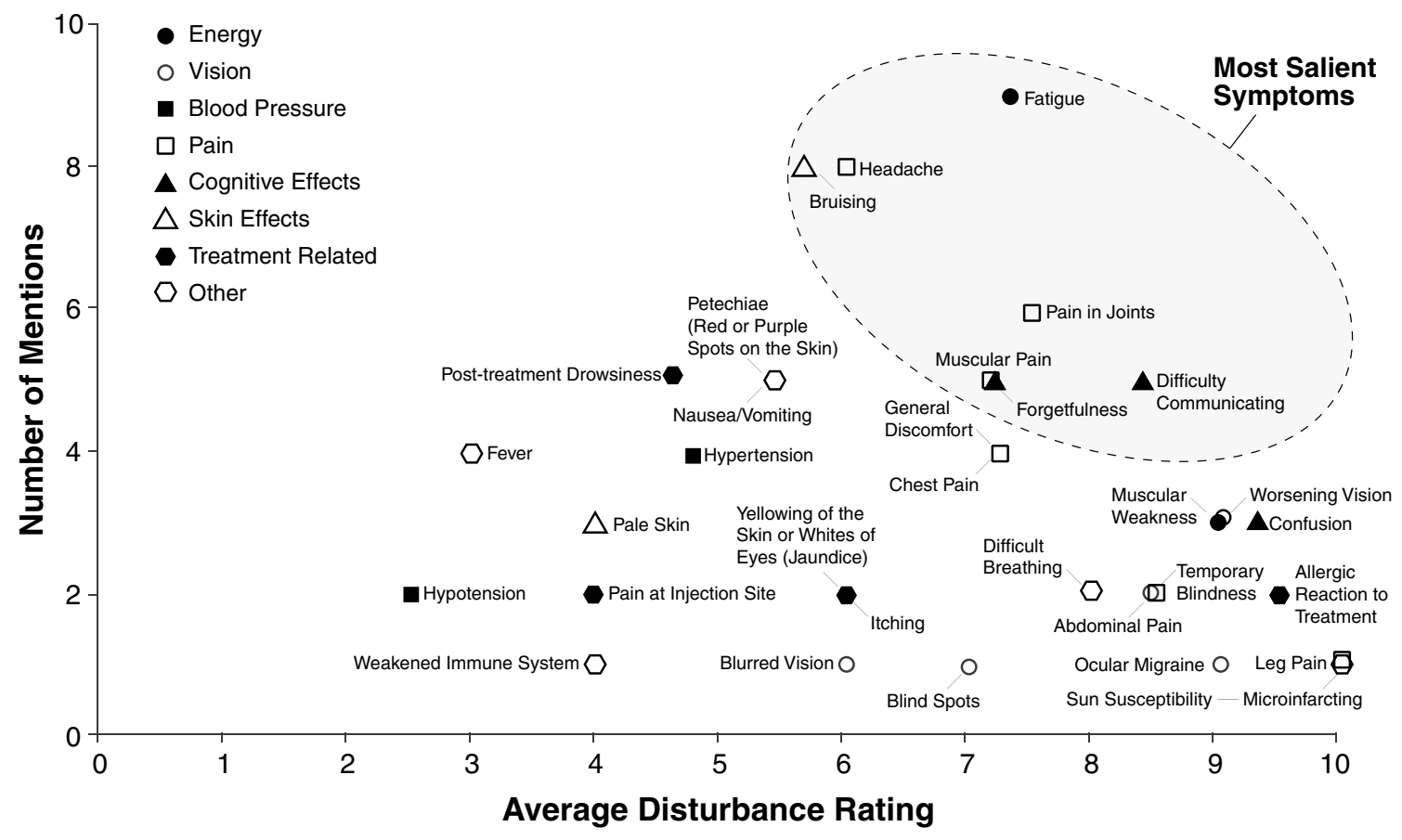

Fig. 2 Symptoms of congenital thrombotic thrombocytopenic purpura: frequency of mention and disturbance ratings by patients

organized by concept code were used to document the elicitation of information by successive interviews. Of a total of 33 symptoms identified, only three (weakened immune system, blind spots, and blurred vision) were newly identified in the final wave of interviews (Table S2 of the Online Appendix). Overall, 27 impacts were identified, three of which (feeling dependent on others, frequent bleeding, spleen removal) emerged in the final wave of interviews (Table S3 of the Online Appendix). As the three newly identified symptoms and three newly identified impacts were mentioned so infrequently by patients, and not hypothesized to be salient from the literature review and clinician interviews, the researchers designated these as minor and not worthy of further interviews to reassess saturation.

An adaptation of grounded theory methods was used to develop the final conceptual model of symptoms, impacts, and treatment-related effects, as shown in Fig. 4. This model incorporated the prior clinical knowledge based on the existing scientific literature and expert opinion to inform the integration of the patient data. From the literature review conducted as the initial part of the process, all concepts were considered in a preliminary conceptual model as necessary and potentially important for describing the patient experience of cTTP. The preliminary conceptual model formed the basis for probing of symptoms and impacts in concept elicitation interviews with clinicians and patients, with the intention of further refining the comprehensiveness and representativeness of the model. All other things being equal, patient input took precedence over other sources in determining which concepts were retained/added to the model, the terminology used to "name" them, and their placement as symptoms vs. immediate impacts vs. general impacts. In addition to the candid descriptions provided by patients, the final conceptual model reflects the disease burden and diminished quality of life experienced by patients with cTTP.

\section{Discussion}

Although the current standard of care for patients with cTTP (i.e., plasma prophylaxis infusions) is effective at reducing the frequency of occurrence of acute episodes, very limited studies have examined its impact on patients' health-related quality of life and long-term outcomes [26]. Generic instruments, such as the HIT-6 (Headache Impact Test), MMSE (Mini-Mental State Examination), and PHQ-8 (Personal Health Questionnaire: Depression Scale), have been used, but they lack content validity in patients with cTTP, and even when combined do not address the full effects of cTTP on patients. We believe this is the first study to provide information on the salient symptoms and impacts of cTTP from patients' perspective and provides a basis for the development of a cTTP-specific PRO tool to assess the disease burden and treatment outcomes in patients.

In this study, we performed a literature review, and interviews with clinicians and patients, to gain a better understanding of the patient journey. Patients provided insight 


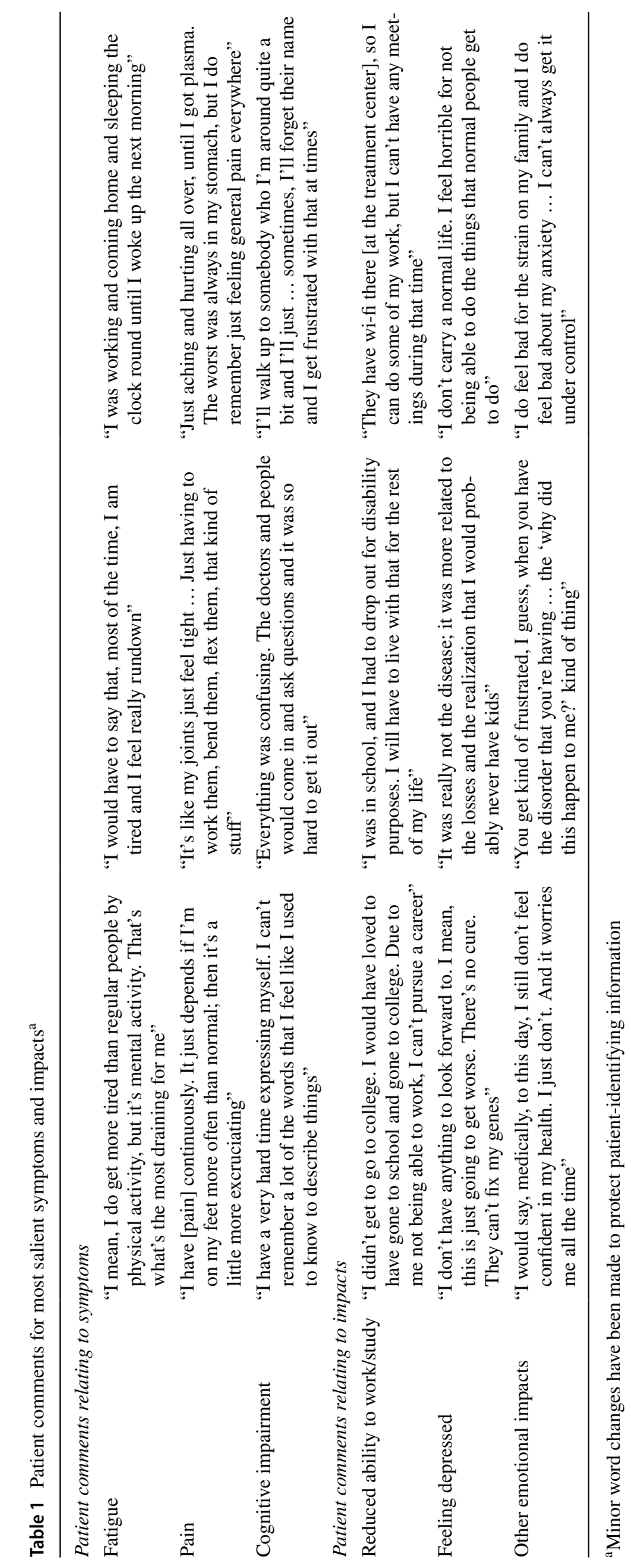




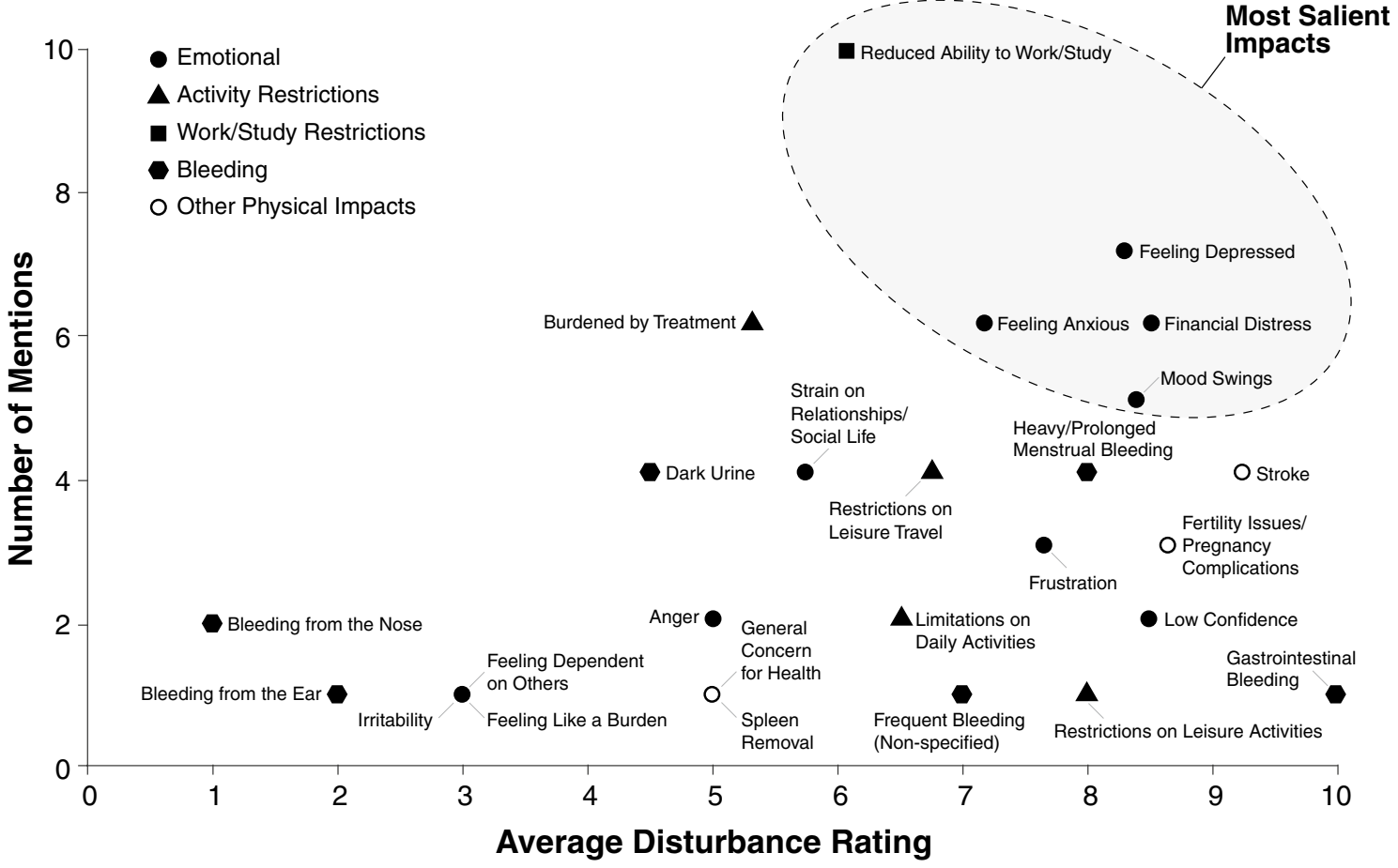

Fig. 3 Impacts of congenital thrombotic thrombocytopenic purpura: frequency of mention and disturbance ratings by patients

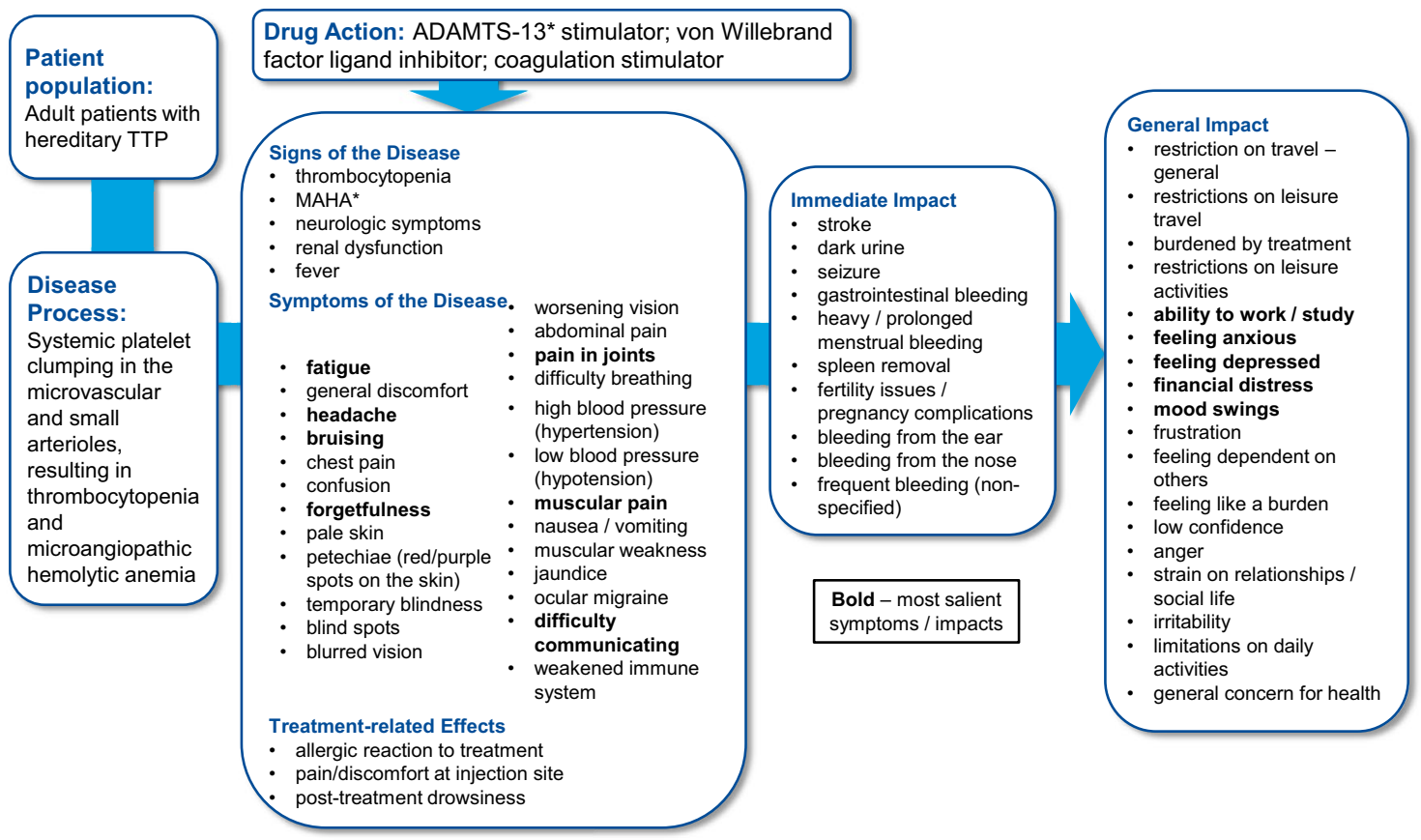

Fig. 4 Final conceptual model for adults with congenital thrombotic thrombocytopenic purpura (TTP). ADAMTS-13 a disintegrin and metalloproteinase with a thrombospondin type 1 motif, member 13, MAHA microangiopathic hemolytic anemia

into their experience of living with cTTP, the concepts that they find most challenging, and the terminology they use to describe the condition. Based on the frequency and degree of associated disturbance; fatigue, cognitive effects (forgetfulness, difficulty communicating), pain (headache, muscle, and joint pain), and bruising emerged as the most salient 
symptoms. Diminished ability to work or study, as well as feelings of anxiety, frustration, and depression, were the concepts with the most salient impact on patients' everyday lives.

Although many of the symptoms and impacts of cTTP identified in the literature review and clinician interviews were similar to those reported by patients, there were notable differences in patients' perceptions of the prominence of those symptoms and impacts. While the literature and clinicians emphasized the more evident impact of travel and treatment burden, patients were more concerned about emotional impacts, such as anxiety, depression, and mood swings, as well as the limitations on their ability to work/ study. With respect to terminology, there were subtle but noticeable differences in the language used. For example, the symptom of confusion was prominent in the literature, but patients used the term "forgetfulness" more frequently.

Although we believe that this body of work provides a better understanding of cTTP from the patients' perspective, the study also has limitations. The involvement of only English-speaking adult US patients with cTTP in the patient interviews may limit the generalizability of the study results beyond the study populations. However, feedback obtained from clinician interviews suggested that the symptoms and impacts of the disease are considerably similar across patients with cTTP regardless of their age or geographical location. The study sample size was small owing to challenges encountered during recruitment, especially with no patient organized group currently existing specifically for cTTP. However, given that the most salient symptoms and impacts all emerged in the first wave of interviews and reached $100 \%$ saturation, as well as their consistency with the literature review and clinician interviews, we are confident that the findings have a high likelihood of being representative across the patient population with cTTP.

Furthermore, we suggest that the framework used to depict the patient experience in this conceptual model for cTTP also could contribute to the development of conceptual models in other rare diseases. This framework can help organize and visualize the key features of the patient population, drug mechanism of action, underlying pathophysiology, and effects of treatment, including both PRO and non-PRO outcomes. In rare diseases, establishing valid and reliable models of the patient experience is especially complicated, given that the heterogeneous nature of these diseases can be expressed quite differently from person to person, or in different subgroups of patients (e.g., age of onset, duration of treatment). The type of conceptual model developed here may then be used to identify and prioritize trial endpoints for potential candidate outcomes of treatment, with consideration given to the unique features of a particular rare disease and its clinical development program.

\section{Conclusions}

The final conceptual model identified the most important symptoms and impacts of the disease and its treatment for patients under the current standard of care for cTTP. Patient-reported outcome instruments are needed to assess the burden of cTTP and evaluate the impact of treatment for improving symptoms and quality of life in patients. However, there is no PRO instrument designed specifically for this purpose. The conceptual model described here adds to our knowledge of the disease from the patients' perspective, and is being used as a framework for developing a cTTPspecific PRO instrument. With a clearer awareness of the patients' experience of cTTP, this instrument could help capture the impact of new treatments on patients' lives.

Acknowledgements Patient recruitment was managed by Sample Czar, Los Angeles, CA, USA. Recruitment assistance was also provided by AnsweringTTP, Toronto, ON, Canada, an international patient advocacy organization. Patient recruitment was funded by Baxalta US Inc., a Takeda company, Lexington, MA, USA. The authors gratefully acknowledge the patients and the following expert clinicians, for participating in interviews: Spero Cataland, Columbus, OH, USA; Kenneth Friedman, Milwaukee, WI, USA; Bernd Jilma, Vienna, Austria; Paul Knoebl, Vienna, Austria; and Marie Scully, London, UK.

Author Contributions AOO, DI, CH, SEB, RNK, and BME were involved in the study design; SEB and RNK were involved in the study conduct; and SEB and RNK were involved in the data analysis and interpretation. All authors were involved in the writing and review of the manuscript. All authors read and approved the final manuscript version submitted for publication.

\section{Compliance with Ethical Standards}

Funding This research was funded by Baxalta US Inc., a member of the Takeda group of companies, Lexington, MA, USA. Medical writing/editorial support for this manuscript was provided by Mark Bowes, PhD, Sharon Suntag, MS, CMPP, and Eric Weathers, BA of IQVIA, Parsippany, NJ, USA, and by Paul Lidbury, PhD, employee of Excel Medical Affairs (Southport, CT, USA) and was funded by Baxalta US Inc., a Takeda company, Lexington, MA, USA. Study management and data analysis assistance were provided by Gabrielle Wilner, MHA of IQVIA, New York, NY, USA.

Conflict of interest Abiola O. Oladapo and Christopher Hibbard are employees of Baxalta US Inc., a Takeda company and Takeda stock owners. Diane Ito was an employee of Baxalta US Inc., a Takeda company at the time this work was conducted and holds stock and stock options. She is now an employee of Stratevi, Boston, MA, USA. Stephanie E. Bean was an employee of IQVIA at the time this work was conducted. She is now an employee of ClearView Healthcare Partners, New York, NY, USA. Robert N. Krupnick is an employee of IQVIA. Bruce M. Ewenstein is an employee of Baxalta US Inc., a Takeda company and holds equity positions.

Data Availability The raw data (transcripts) of this study cannot be shared, to maintain participant privacy. Summary data are available from the corresponding author on reasonable request. 
Open Access This article is distributed under the terms of the Creative Commons Attribution-NonCommercial 4.0 International License (http://creativecommons.org/licenses/by-nc/4.0/), which permits any noncommercial use, distribution, and reproduction in any medium, provided you give appropriate credit to the original author(s) and the source, provide a link to the Creative Commons license, and indicate if changes were made.

\section{References}

1. Blombery P, Scully M. Management of thrombotic thrombocytopenic purpura: current perspectives. J Blood Med. 2014;5:15-23.

2. Galbusera M, Noris M, Remuzzi G. Inherited thrombotic thrombocytopenic purpura. Haematologica. 2009;94(2):166-70.

3. Orphanet. Congenital thrombotic thrombocytopenic purpura. http://www.orpha.net/consor/cgi-bin/Disease_Searc h.php? Ing=EN\&data_id=12422\&Disease_Disease_Search_disea seGroup=upshaw-schulman\&Disease_Disease_Search_disea seType $=$ Pat $\&$ Disease $(\mathrm{s}) /$ group $\% 20$ of $\% 20$ diseases $=$ Congenital -thrombotic-thrombocytopenic-purpura\&title $=$ Congenital-throm botic-thrombocytopenic-purpura\&search=Disease_Search_Simpl e. Accessed 6 Nov 2017.

4. George JN, Nester CM. Syndromes of thrombotic microangiopathy. N Engl J Med. 2014;371(7):654-66.

5. Scully M, Cataland S, Coppo P, de la Rubia J, Friedman KD, Kremer Hovinga J, et al. Consensus on the standardization of terminology in thrombotic thrombocytopenic purpura and related thrombotic microangiopathies. J Thromb Haemost. 2016;15(2):312-22.

6. Wun TW. Thrombotic thrombocytopenic purpura (TTP). http:// emedicine.medscape.com/article/206598-followup\#e5. Accessed 3 Sep 2017.

7. Kremer Hovinga JA, Coppo P, Lämmle B, Moake JL, Miyata T, Vanhoorebeke K. Thrombotic thrombocytopenic purpura. Nat Rev. 2017;3:1-17.

8. Knobl P. Inherited and acquired thrombotic thrombocytopenic purpura (TTP) in adults. Semin Thromb Hemost. 2014;40(4):493-502.

9. George JN. Hereditary thrombotic thrombocytopenic purpura (TTP). http://www.uptodate.com/contents/hereditary-throm botic-thrombocytopenic-purpura-ttp?source $=$ search_resul t\&search $=$ thrombotic + thrombocytopenic + purpura\&selectedTi tle $=2 \% 7 \mathrm{E} 150$. Accessed 18 Feb 2018 .

10. George JN, Cuker A. Acquired TTP: clinical manifestations and diagnosis. http://www.uptodate.com/contents/acquiredttp-clinical-manifestations-and-diagnosis?source=search_resul $\mathrm{t} \&$ search $=$ thrombotic + thrombocytopenic + purpura\&selectedTi tle $=1 \sim 150$. Accessed 1 Feb 2016.

11. Patrick DL, Burke LB, Gwaltney CJ, Leidy NK, Martin ML, Molsen E, Ring L. Content validity: establishing and reporting the evidence in newly developed patient-reported outcomes (PRO) instruments for medical product evaluation. ISPOR PRO Good Research Practices Task Force report: part 1: eliciting concepts for a new PRO instrument. Value Health. 2011;14(8):967-77.
12. Patrick DL, Burke LB, Gwaltney CJ, Leidy NK, Martin ML, Molsen E, et al. Content validity: establishing and reporting the evidence in newly developed patient-reported outcomes (PRO) instruments for medical product evaluation. ISPOR PRO Good Research Practices Task Force report: part 2: assessing respondent understanding. Value Health. 2011;14(8):978-88.

13. US Food and Drug Administration. Guidance document for industry, December 2009. Patient-reported outcome measures: use in medical product development to support labeling claims. https:// www.fda.gov/regulatory-information/search-fda-guidance-docum ents/patient-reported-outcome-measures-use-medical-productdevelopment-support-labeling-claims. Accessed 22 July 2019.

14. Muhr T. User's manual for ATLAS.ti 5.0. Berlin: ATLAS.ti Scientific Software Development GmbH; 2004.

15. Thrombotic thrombocytopenic purpura. https://www.nlm.nih.gov/ medlineplus/ency/article/000552.htm. Accessed 1 Jun 2019.

16. Thrombotic thrombocytopenic purpura: practice essentials, pathophysiology, background. http://emedicine.medscape.com/artic le/206598-overview\#showall. Accessed 26 Jan 2016.

17. Deford CC, Reese JA, Schwartz LH, Perdue JJ, Kremer Hovinga JA, Lammle B, et al. Multiple major morbidities and increased mortality during long-term follow-up after recovery from thrombotic thrombocytopenic purpura. Blood. 2013;122(12):2023-9.

18. George JN. How I treat patients with thrombotic thrombocytopenic purpura: 2010. Blood. 2010;116(20):4060-9.

19. George JN, Al-Nouri ZL. Diagnostic and therapeutic challenges in the thrombotic thrombocytopenic purpura and hemolytic uremic syndromes. Hematol Am Soc Hematol Educ Program. 2012;2012:604-9.

20. Hanby HA, Zheng XL. Current status in diagnosis and treatment of hereditary thrombotic thrombocytopenic purpura. Hered Genet. 2014;3(1):e108.

21. Kennedy AS, Lewis QF, Scott JG, Kremer Hovinga JA, Lammle B, Terrell DR, et al. Cognitive deficits after recovery from thrombotic thrombocytopenic purpura. Transfusion. 2009;49(6):1092-101.

22. National Heart, Lung, and Blood Institute, National Institutes of Health. What is thrombotic thrombocytopenic purpura? https:// www.nhlbi.nih.gov/health/health-topics/topics/ttp. Accessed 26 Jan 2016.

23. Scully M, Hunt BJ, Benjamin S, Liesner R, Rose P, Peyvandi F, et al. Guidelines on the diagnosis and management of thrombotic thrombocytopenic purpura and other thrombotic microangiopathies. Br J Haematol. 2012;158(3):323-35.

24. Fujimura Y, Matsumoto M, Isonishi A, Yagi H, Kokame K, Soejima K, et al. Natural history of Upshaw-Schulman syndrome based on ADAMTS13 gene analysis in Japan. J Thromb Haemost. 2011;9(Suppl. 1):283-301.

25. Lotta LA, Wu HM, Mackie IJ, Noris M, Veyradier A, Scully MA, et al. Residual plasmatic activity of ADAMTS13 is correlated with phenotype severity in congenital thrombotic thrombocytopenic purpura. Blood. 2012;120(2):440-8.

26. Mansouri Taleghani M, von Krogh AS, Fujimura Y, George JN, Hrachovinova I, Knobl PN, et al. Hereditary thrombotic thrombocytopenic purpura and the hereditary TTP registry. Hamostaseologie. 2013;33(2):138-43. 